\title{
FACTORS AFFECTING REPRODUCTIVE AND PRODUCTIVE EFFICIENCY OF NILI RAVI BUFFALOES (Bubablus Bubalis) IN PUNJAB, PAKISTAN
}

\author{
M. A. Yasir ${ }^{*}$, A. Sattar ${ }^{2}$, M. Z. Tahir ${ }^{2}$, M. Akhtarr ${ }^{1}$, M. Binyameen ${ }^{1}$ and Hammad-ur-Rehman ${ }^{3}$ \\ ${ }^{* 1}$ Buffalo Research Institute (BRI), Pattoki, District Kasur \\ ${ }^{2}$ Department of Theriogenology, University of Veterinary and Animal Sciences, Lahore, Pakistan \\ ${ }^{3}$ University Diagnostic Lab, University of Veterinary \& Animal Sciences, Lahore, Pakistan \\ ${ }^{*}$ Corresponding author's email: ammaryasir348@gmail.com \\ The paper was presented in International Buffalo Congress 2019, February 18-20, Lahore, Pakistan
}

\begin{abstract}
The data on Nili Ravi buffaloes kept at Buffalo Research Institute (BRI), Pattoki, District Kasur, Punjab Pakistan were collected from 2010 to 2018 to estimate the factors affecting the reproductive and productive traits of Nili Ravi buffaloes. The reproductive parameters including age at maturity, age at first conception, age at first calving, gestation length, service period, calving interval and number of services per conception were $1029.59 \pm 20.60$ days, $1065.33 \pm$ 20.20 days, $1417.6 \pm 21.66$ days, $312.27 \pm 8.03$ days, $227.16 \pm 4.98$ days, $485.15 \pm 3.80$ days and $1.60 \pm 0.02$, respectively. The productive parameters including 305-day milk yield, lactation length and dry period were $2211.05 \pm$ 20.06 liters, $266.94 \pm 1.49$ days and $279.47 \pm 5.19$ days, respectively. The statistical analysis for the comparison among different treatments ANOVA technique was employed using GLM procedures. A significant effect of year and season of birth on age at maturity, age at first conception and age at first calving was observed $(\mathrm{P} \leq 0.05)$. The year of calving, season of calving and parity significantly affected calving interval, service period, services per conception and 305-day milk yield $(\mathrm{P} \leq 0.05)$. It is concluded that improvement in performance of Nili Ravi buffaloes can be made by better management, nutrition and effective heat detection program. Improved management of heifers may reduce age at maturity and age at first calving. Culling on the basis of production performance may improve productivity of buffalo in future.
\end{abstract}

Key words: Pakistan, Nili-Ravi Buffalo, productive traits, reproductive traits. https://doi.org/10.36899/JAPS.2020.3.0067

Published online March 25, 2020

\section{INTRODUCTION}

The livestock sector plays an important position in the economy of Pakistan. This sector has a $11.1 \%$ share in national GDP and $58.9 \%$ share in the agriculture GDP and showed increase of $3.8 \%$ in gross value addition during year 2017-2018. The population of cattle is higher than buffalos in Pakistan (46.1 VS 38.8 million) but contribution of buffalo is more than cow (60.69 VS $36.11 \%$ ) towards total milk production of country (Anonymous 2018). Nili-Ravi is the most popular breed of buffaloes which constitutes about $76.7 \%$ of the total buffalo population in Pakistan (Garcia et al. 2016).

Buffalo (Bubalus bubalis) is main dairy animal in Pakistan and second most important dairy species in the world. The performance traits of buffaloes are influenced by several factors. These factors are year and season of birth, year and season of calving and parity. The true genetic merit of the animals may be masked by these factors. The study of these factors affecting performance traits of Nili-Ravi buffaloes is therefore very important for genetic evaluations of animals, developing breeding and general management strategies/programs. Mostly buffaloes are owned by small landless farmers keeping 2-3 animals only. Buffalo population of Pakistan is an important national asset comprising the best dairy breeds of the world, i.e., Nili Ravi and Kundi (Akhtar et al. 2006). Pakistani buffaloes have the ability to produce more than 5000 liter of milk per lactation under wellorganized breeding, feeding and health care plan (Bilal et al., 2006). There are many constraints being faced by the dairymen, which can be classified as genetic and environmental (Elemam and Nekheila 2012). Higher age at first calving and long calving interval are not beneficial to the dairy producers. Calving interval, age at maturity, services per conception and birth weight are most important traits to gauge the farm economy. A farm with 15 -month calving interval, 1.33 services per conception could be inexpensively advantageous. So it is imperative to assess the different productive and reproductive performance parameters like age at maturity, age at first conception, age at first calving, number of services or A.I per conception, service period, calving interval, lactation length, milk yield and dry period.

The traits of greater importance in buffalo are fertility, milk production, fat production and productive life span. All these traits are dependent upon the reproductive efficiency of buffaloes. Cady et al. (1983) 
evaluated performance of Nili-Ravi breed at two institutional herds and reported that herd, year and season of calving and parity affected $(\mathrm{P} \leq 0.01)$ productive and reproductive traits (milk yield, lactation length, days open, calving interval and services per conception). Naqvi (2000) included six military dairy farms to study the effect of parity and season of calving on service period in Nili-Ravi buffalo and reported reduction in length of service period with the increase in parity and significantly lower service period in spring and winter calvers as compared to summer and fall.

The objective of this study was to assess the parameters of reproductive and productive efficiency like; age at maturity, age at first conception, age at first calving, calving interval, service period, number of services per conception, gestation length, lactation length, dry period, milk yield and breeding efficiency in NiliRavi buffaloes maintained at BRI, Pattoki.

\section{MATERIALS AND METHODS}

The parameters of reproductive and productive efficiency of Nili Ravi buffaloes kept at Buffalo Research Institute (BRI), Pattoki, District Kasur, Punjab Pakistan $\left(31^{\circ} 1^{\prime} \mathbf{3 0 . 0 3 2 4} " \mathrm{~N}\right.$ and $73^{\circ} 50^{\prime} \mathbf{5 2 . 3 6 0 8 "}$ E.) during 2010 2018 were studied. Data relating to reproductive and productive performance of Nili Ravi buffaloes at BRI were collected from history sheets for year 2010 to 2018 .

During 2005-2006 Buffalo Research Institute, Pattoki District Kasur was established. At that time total of 400 Nili Ravi buffaloes were purchased from local breeders to be maintained at LES, Bhunikey. Nili Ravi buffaloes were kept in loose housing system with ad libitum supply of water. Buffaloes were also fed concentrate ration according to the body requirement based on production status along with green fodder and roughages. The buffalo herd was provided with adequate watering and bathing facilities throughout the year. All the buffaloes were inseminated with frozen thawed semen when they exhibited estrus. Teaser bulls were kept in the herd for detection of estrus in buffaloes. The activity of the teaser bull was observed by the experienced stockman.

The information was recorded from history / pedigree sheets on following aspects.

Identification number of buffalo, date of [birth, first insemination, successful service/ A.I, calving and drying], status of calving, total number of calving (lactation) and milk yield per lactation. These data were used to evaluate the factors affecting the following parameters pertaining to reproductive and productive efficiency of buffaloes like; Age at maturity, age at first conception, age at first calving, calving interval, service period, gestation length, service / A.I per conception, lactation length, dry period, 305-day milk yield and breeding efficiency.
Incomplete lactation record of $\leq 181$ days due to culling, abortion, premature birth or diseases, etc were excluded from the data after Chaudhry (1992). The records of the animals with calving interval $<300$ or $>730$ days, gestation length $<285$ or $>335$ days, age at maturity $>1825$ days, age at first conception $>2190$ days, age at first calving $<900$ or $>2400$ days were also excluded (Cady et al. 1983).

To determine the effect of season on different parameters, the month of year were divided into five seasons as reported by Ali et al. (2011).

I. Spring_ February, March and April

II. Dry Summer_ May and June

III. Humid Summer July, Aug and Sep

IV. Autumn October and November

V. Winter_December and January

The statistical model ANOVA employed using GLM procedures to analyze the productive and reproductive traits affected by different factors ie. year and season of birth/calving and parity. Significant treatment means were separated through SNK test.

Breeding Efficiency: Breeding efficiency is measure of capacity to reproduce by adult farm stock. Breeding efficiency or reproductive efficiency of Nili Ravi buffaloes on the basis of calving interval of 400 days had been calculated through formula by Sharma et al. (1980).

$$
[900+(n-1) 400] \times 100
$$

B.E $(\%)=$

$$
\text { AFC (Days) + CI (Days) }
$$

Where

$\mathrm{n}$ is the number of calvings or parturitions, 400 is the ideal calving interval in buffaloes.

Statistical Analysis: Descriptive statistics was employed in the form of averages and percentages along with appropriate graph type. For the comparison among different treatments ANOVA technique was employed using GLM procedures in SAS software (Version 9.1.). Significant treatment means were separated through SNK test.

\section{RESULTS}

Effect of year of birth/calving: In this study, the data analysis of Nili Ravi buffaloes revealed that age at maturity, age at first conception, age at first calving, calving interval, service period, gestation length, number of service per conception, milk yield, lactation length and dry period (mean \pm SE) averaged $1029.59 \pm 20.60$, $1056.46 \pm 20.41,1417.06 \pm 21.66,490.85 \pm 3.82,227.16$ $\pm 4.98,312.27 \pm 8.03$ days, $1.60 \pm 0.29$ numbers, 1917.07 \pm 15.16 liters, $266.94 \pm 1.49$ and $279.47 \pm 5.19$ days, respectively (Table 1). Year of birth have significant $(\mathrm{P} \leq$ 0.05 ) effect on age at maturity, age at first conception and age at first calving among the buffaloes born during different years (Table 2). 
Year of calving have significant $(\mathrm{P} \leq 0.05)$ effect on calving interval, service period and 305-day milk yield among the buffaloes calving during different years (Table 5). Gestation length, lactation length and dry period were not affected by years (Table 5).

Effect of season of birth/calving: Season of birth have significant $(P \leq 0.05)$ effect on age at maturity, age at first conception and age at first calving. The maximum $(1216.10 \pm 56.02$ days $)$ age at maturity was found in buffalo heifers born during winter and the minimum (942.74 \pm 31.02 days) was found in autumn born heifers (Table 3). Age at first conception maximum (1221.43 \pm 60.57 days) and the minimum ( $950.07 \pm 33.03$ days) was found in buffalo heifers born during winter and autumn, respectively (Table 3). Age at first calving maximum (1568.45 \pm 64.31 days) was found in buffaloes born during winter season and minimum $(1269.58 \pm 40.67$ days) was found in autumn born heifers (Table 3). Autumn born calvers have plenty of good quality fodder available during their early days of age (3-6 months) which may have helped them to growth rate in later period even in low quality fodder.

Season of calving have significant $(\mathrm{P} \leq 0.05)$ effect on calving interval, service period, number of services per conception, 305-day milk yield, lactation length and dry period. The average calving interval for winter calvers was the longest ( $532.43 \pm 11.23$ days). The shortest ( $450.94 \pm 14.39$ days) calving interval was found in Nili Ravi buffaloes calving during humid summer (Table 6). The possible reason may be seasonality of calving in buffaloes as winter calvers wait longer to rebreed as compared to summer calvers. Better management and feeding during winter season can reduce the calving interval. The average service period for spring calvers was the longest $(249.95 \pm 22.06$ days $)$. The shortest (450.94 \pm 14.39 days) service period was found in Nili Ravi buffaloes calving during humid summer. The maximum $(1.86 \pm 0.12)$ and the minimum $(1.41 \pm 0.06)$ services per conception were found in buffaloes calving during dry summer and autumn, respectively (Table 6).

The season affected 305-day milk yield, the maximum (2352.56 \pm 45.67 liters) was found in spring calvers and the minimum $(2159.32 \pm 19.44$ liters $)$ in summer calvers (Table 6). Poor milk yield in summer calvers than spring calvers may be due to high temperature and low availability of fodder. Season of calving influenced lactation length, the maximum (277.77 \pm 3.90 days) lactation length was found in autumn calvers and minimum $(254.63 \pm 6.72$ days $)$ in dry summer calvers (Table 6). Lower lactation length is mainly due to insufficiency of fodder in summer season. Season of calving influenced dry period, the maximum (317.06 \pm
19.88 days) dry period was found in spring calvers and minimum (234.62 \pm 13.20 days) in autumn calvers, (Table 6). The shorter dry period in autumn may be attributed to longer lactation length of buffaloes. The gestation length is less studied trait in buffaloes. Season did not affect it (Table 6).

Effect of Parity: The effect of parity/lactation number on calving interval, service period, number of services per conception, 305-day milk yield and dry period was found to be significant $(\mathrm{P} \leq 0.05)$. The highest $(537.52 \pm 8.02$ days) and the lowest $(434.44 \pm 36.67$ days $)$ calving interval was observed in $1^{\text {st }}$ lactation and $8^{\text {th }}$ lactation, respectively (Table 4) showing gradual decrease from first to later parities. The calving interval has two components i.e. lactation length and dry period. The increased calving interval may be due to high dry period. The lactation length was not affected by parity in this study so dry period decreased along with parity which reduces the calving interval in later ages. The service period, highest (297.84 \pm 9.59 days) and the lowest $(157.51 \pm 28.16$ days $)$ was observed in $1^{\text {st }}$ lactation and $7^{\text {th }}$ lactation, respectively (Table 4) showing gradual decrease from first to later parities. First calvers suffer more from stress due to growing, producing and insufficient energy available to them, so there may be difficulty for early conception in first calvers. The service period and gestation length are two component of calving interval. Only service period can be altered to reduce the calving interval. The parity affected number of services per conception. The services per conception were more in earlier parities as compared to the later parities (Table 4).

Significant effect of parity on 305-day milk yield was observed, the highest $(2243.79 \pm 34.25$ liters $)$ and the lowest $(2005.44 \pm 101.30$ liters $)$ milk yield was observed in $2^{\text {nd }}$ lactation and $8^{\text {th }}$ lactation, respectively (Table 4). Parity affected dry period, the highest (349.90 \pm 14.51 days) dry period was found in first calvers, it decreased with increased in parity and reached to the lowest (203.54 \pm 33.21 days) in $7^{\text {th }}$ lactation (Table 4). The longer dry period was due to poor lactation yield in first parity buffaloes.

The effect of parity on gestation length and lactation length found to be non-significant $(\mathrm{P}>0.05)$. The gestation length was not affected by parity (Table 4 ). Parity did not affect lactation length, the lactation length was highest $(277.17 \pm 4.88$ days $)$ in second calvers, it decreased with increased in parity and reached to the lowest $\left(243.33 \pm 13.91\right.$ days) in $8^{\text {th }}$ lactation (Table 4$)$.

Breeding Efficiency: The data analysis of 250 Nili Ravi buffaloes revealed that the breeding efficiency is $86.45 \%$ (Table 7). 
Table 1. Reproductive and Productive traits (Mean \pm SE) in Nili Ravi Buffaloes

\begin{tabular}{lcc}
\hline $\begin{array}{l}\text { Reproductive and Productive } \\
\text { Performance Parameter }\end{array}$ & No. of Observations & Overall Mean \pm SE \\
\hline Age at Maturity (Days) & 192 & $1029.59 \pm 20.60$ \\
Age at first Conception (Days) & 191 & $1056.46 \pm 20.41$ \\
Age at first Calving (Days) & 218 & $1417.06 \pm 21.66$ \\
Calving Interval (Days) & 855 & $490.85 \pm 3.82$ \\
Service Period (Days) & 1074 & $227.16 \pm 4.98$ \\
Gestation Length (Days) & 1553 & $312.27 \pm 8.03$ \\
Services Per Conception (No.) & 1716 & $1.60 \pm 0.29$ \\
305- day Milk Yield (Liters) & 1117 & $2211.05 \pm 20.06$ \\
Lactation Length (Days) & 1146 & $266.94 \pm 1.49$ \\
Dry Period (Days) & 940 & $279.47 \pm 5.19$ \\
\hline
\end{tabular}

Table 2. Effect of Year of Birth on Age at Maturity, Age at first Conception and Age at first Calving (Mean \pm SE) in Nili Ravi Buffaloes

\begin{tabular}{|c|c|c|c|}
\hline Year of Birth & Age at Maturity (Days) & Age at first Conception (Days) & Age at first Calving (Days) \\
\hline \multirow[t]{2}{*}{2010} & $1270.10 \pm 29.25^{\mathrm{ab}}$ & $1275.84 \pm 31.10^{\mathrm{ab}}$ & $1590.71 \pm 31.12^{\mathrm{b}}$ \\
\hline & $(46)$ & $(47)$ & $(57)$ \\
\hline \multirow[t]{2}{*}{2011} & $1220.96 \pm 44.52^{\mathrm{ab}}$ & $1220.9 \pm 47.40^{\mathrm{b}}$ & $1528.69 \pm 46.11^{\mathrm{bc}}$ \\
\hline & $(42)$ & $(42)$ & $(58)$ \\
\hline \multirow[t]{2}{*}{2012} & $1340.21 \pm 68.60^{\mathrm{a}}$ & $1353.92 \pm 73.05^{\mathrm{a}}$ & $1715.61 \pm 84.80^{\mathrm{a}}$ \\
\hline & $(15)$ & $(15)$ & $(24)$ \\
\hline \multirow[t]{2}{*}{2013} & $1075.13 \pm 55.15^{\mathrm{b}}$ & $1108.31 \pm 58.72^{c}$ & $1419.01 \pm 62.41^{\mathrm{c}}$ \\
\hline & (19) & (19) & $(23)$ \\
\hline \multirow[t]{2}{*}{2014} & $951.96 \mathrm{~b} \pm 54.21^{\mathrm{bc}}$ & $960.63 \pm 57.65^{d}$ & $1238.67 \pm 57.84^{\mathrm{d}}$ \\
\hline & $(26)$ & $(27)$ & $(29)$ \\
\hline \multirow[t]{2}{*}{2015} & $759.14 \pm 35.45^{\mathrm{c}}$ & $798.96 \pm 40.01^{\mathrm{e}}$ & $1060.13 \pm 54.57^{\mathrm{e}}$ \\
\hline & $(40)$ & $(37)$ & $(21)$ \\
\hline \multirow[t]{2}{*}{2016} & $570.83 \pm 98.43^{\mathrm{d}}$ & $634.16 \pm 104.81^{\mathrm{f}}$ & $1001.65 \pm 88.43^{\mathrm{f}}$ \\
\hline & $(4)$ & $(4)$ & $(6)$ \\
\hline
\end{tabular}

Table 3. Effect of Season of Birth on Age at Maturity, Age at first Conception and Age at first Calving (Mean \pm SE) in Nili Ravi Buffaloes

\begin{tabular}{|c|c|c|c|}
\hline Season of Birth & Age at Maturity (Days) & $\begin{array}{c}\text { Age at first Conception } \\
\text { (Days) }\end{array}$ & $\begin{array}{c}\text { Age at first Calving } \\
\text { (Days) }\end{array}$ \\
\hline Winter & $\begin{array}{c}1216.10 \pm 56.02^{a} \\
(16)\end{array}$ & $\begin{array}{c}1221.43 \pm 60.57^{\mathrm{a}} \\
(14)\end{array}$ & $\begin{array}{c}1568.45 \pm 64.31^{\mathrm{a}} \\
(27)\end{array}$ \\
\hline Spring & $\begin{array}{c}1086.57 \pm 48.66^{b} \\
(22)\end{array}$ & $\begin{array}{c}1130.43 \pm 52.13^{b} \\
(21)\end{array}$ & $\begin{array}{c}1499.21 \pm 56.08^{b} \\
(29)\end{array}$ \\
\hline Dry Summer & $\begin{array}{c}1116.19 \pm 52.09^{b} \\
(19)\end{array}$ & $\begin{array}{c}1116.19 \pm 55.47^{b} \\
(19)\end{array}$ & $\begin{array}{c}1452.86 \pm 62.77^{b} \\
(28)\end{array}$ \\
\hline Humid Summer & $\begin{array}{c}959.51 \pm 21.33^{c} \\
(92)\end{array}$ & $\begin{array}{c}990.07 \pm 33.03^{c} \\
(94)\end{array}$ & $\begin{array}{c}1342.79 \pm 23.62^{c} \\
(85)\end{array}$ \\
\hline Autumn & $\begin{array}{c}942.74 \pm 31.02^{c} \\
(43)\end{array}$ & $\begin{array}{c}950.07 \pm 33.03^{d} \\
(43)\end{array}$ & $\begin{array}{c}1269.58 \pm 40.67^{\mathrm{d}} \\
(49)\end{array}$ \\
\hline
\end{tabular}


Table 4. Effect of Parity on Reproductive and Productive traits (Mean \pm SE) in Nili Ravi Buffaloes

\begin{tabular}{|c|c|c|c|c|c|c|}
\hline Parity & $\begin{array}{l}\text { Services/ } \\
\text { Conception }\end{array}$ & Service Period & $\begin{array}{l}\text { Calving } \\
\text { Interval }\end{array}$ & $\begin{array}{l}\text { 305-day Milk } \\
\text { Yield }\end{array}$ & $\begin{array}{l}\text { Lactation } \\
\text { Length }\end{array}$ & Dry Period \\
\hline 1 & $\begin{array}{l}1.24 \pm 0.06 \\
(357)\end{array}$ & $\begin{array}{c}297.84 \pm 9.59^{\mathrm{a}} \\
(267)\end{array}$ & $\begin{array}{c}537.52 \pm 8.02^{\mathrm{a}} \\
(188)\end{array}$ & $\begin{array}{c}2193.75 \pm 28.36^{\mathrm{ab}} \\
(271)\end{array}$ & $\begin{array}{c}276.02 \pm 4.39 \\
(278)\end{array}$ & $\begin{array}{c}349.90 \pm 14.51^{\mathrm{a}} \\
(238)\end{array}$ \\
\hline 2 & $\begin{array}{l}1.42 \pm 0.07 \\
\quad(261)\end{array}$ & $\begin{array}{c}253.49 \pm \\
11.56^{\mathrm{b}} \\
(184)\end{array}$ & $\begin{array}{c}513.16 \pm 9.13^{\mathrm{ab}} \\
(145)\end{array}$ & $\begin{array}{c}2243.79 \pm 34.25^{\mathrm{a}} \\
(182)\end{array}$ & $\begin{array}{l}277.17 \pm 4.88 \\
\quad(185)\end{array}$ & $\begin{array}{c}294.46 \pm \\
14.10^{\mathrm{b}} \\
(160)\end{array}$ \\
\hline 3 & $\begin{array}{l}1.73 \pm 0.07 \\
\quad(231)\end{array}$ & $\begin{array}{c}220.06 \pm 12.89^{c} \\
(148)\end{array}$ & $\begin{array}{c}486.18 \pm \\
10.04^{\mathrm{b}} \\
(120)\end{array}$ & $\begin{array}{c}2178.26 \pm 38.23^{\mathrm{ab}} \\
(155)\end{array}$ & $\begin{array}{l}269.13 \pm 5.01 \\
\quad(158)\end{array}$ & $\begin{array}{c}282.04 \pm \\
16.74^{\mathrm{b}} \\
(131)\end{array}$ \\
\hline 4 & $\begin{array}{l}1.93 \pm 0.07 \\
\quad(262)\end{array}$ & $\begin{array}{c}195.14 \pm \\
12.47^{\mathrm{d}} \\
(158)\end{array}$ & $\begin{array}{c}462.98 \pm 9.29^{b} \\
(140)\end{array}$ & $\begin{array}{c}2137.77 \pm 35.94^{b} \\
(182)\end{array}$ & $\begin{array}{l}259.31 \pm 5.59 \\
(185)\end{array}$ & $\begin{array}{c}252.39 \pm \\
20.25^{\mathrm{bc}} \\
(151)\end{array}$ \\
\hline 5 & $\begin{array}{l}1.78 \pm 0.08 \\
(216)\end{array}$ & $\begin{array}{c}173.68 \pm 13.59^{\mathrm{e}} \\
(133)\end{array}$ & $\begin{array}{c}451.96 \pm 9.84^{\mathrm{bc}} \\
(125)\end{array}$ & $\begin{array}{c}2080.79 \pm 38.11^{b c} \\
(153)\end{array}$ & $\begin{array}{c}264.20 \pm 6.26 \\
(156)\end{array}$ & $\begin{array}{c}239.42 \pm 21.00^{c} \\
(131)\end{array}$ \\
\hline 6 & $\begin{array}{l}1.74 \pm 0.09 \\
\quad(162)\end{array}$ & $\begin{array}{c}165.79 \pm 16.91^{\mathrm{e}} \\
(86)\end{array}$ & $\begin{array}{c}447.96 \pm \\
12.07^{\mathrm{bc}} \\
(83)\end{array}$ & $\begin{array}{c}2109.83 \pm 44.12^{\mathrm{b}} \\
(109)\end{array}$ & $\begin{array}{l}261.28 \pm 6.89 \\
\quad(113)\end{array}$ & $\begin{array}{c}233.04 \pm 22.94^{\mathrm{c}} \\
(86)\end{array}$ \\
\hline 7 & $\begin{array}{l}1.47 \pm 0.12 \\
(92)\end{array}$ & $\begin{array}{c}157.51 \pm 28.16^{\mathrm{e}} \\
(31)\end{array}$ & $\begin{array}{c}461.85 \pm \\
18.86^{\mathrm{b}} \\
(34)\end{array}$ & $\begin{array}{c}2051.42 \pm 65.55^{\mathrm{c}} \\
(50)\end{array}$ & $\begin{array}{c}261.72 \pm 8.88 \\
\quad(55)\end{array}$ & $\begin{array}{c}203.54 \pm \\
33.21^{\mathrm{d}} \\
(32)\end{array}$ \\
\hline 8 & $\begin{array}{l}1.24 \pm 0.20 \\
\quad(33)\end{array}$ & $\begin{array}{l}182.12 \pm \\
55.44^{\text {de }} \\
(8)\end{array}$ & $\begin{array}{c}434.44 \pm 36.67^{\mathrm{c}} \\
(9)\end{array}$ & $\begin{array}{c}2005.44 \pm 101.30^{\mathrm{d}} \\
(14)\end{array}$ & $\begin{array}{c}243.33 \pm 13.91 \\
(14)\end{array}$ & $\begin{array}{c}213.50 \pm \\
52.70^{\text {cd }} \\
(9)\end{array}$ \\
\hline
\end{tabular}

The values with different superscript in each column differ significantly $(P \leq 0.05)$ but the values sharing at least one superscript in each column did not differ significantly $(P \leq 0.05)$.

Table 5. Effect of Year of Calving on Reproductive and Productive traits (Mean \pm SE) in Nili-Ravi Buffaloes

\begin{tabular}{|c|c|c|c|c|c|c|c|}
\hline $\begin{array}{l}\text { Year of } \\
\text { Calving }\end{array}$ & $\begin{array}{l}\text { Service } \\
\text { Period }\end{array}$ & $\begin{array}{l}\text { Gestation } \\
\text { Length }\end{array}$ & $\begin{array}{l}\text { Calving } \\
\text { Interval }\end{array}$ & $\begin{array}{l}\text { Lactation } \\
\text { Length }\end{array}$ & $\begin{array}{l}\text { Dry } \\
\text { Period }\end{array}$ & $\begin{array}{l}\text { 305-day Milk } \\
\text { Yield }\end{array}$ & $\begin{array}{l}\text { Services } \\
\text { per } \\
\text { Conception }\end{array}$ \\
\hline 2010 & $\begin{array}{c}221.28 \pm \\
12.03^{\mathrm{ab}} \\
(232)\end{array}$ & $\begin{array}{c}314.74 \pm 0.82 \\
(242)\end{array}$ & $\begin{array}{c}481.53 \pm \\
8.73^{\mathrm{ab}} \\
(198)\end{array}$ & $\begin{array}{c}257.22 \pm \\
5.38 \\
(208)\end{array}$ & $\begin{array}{c}303.21 \pm \\
15.78 \\
(222)\end{array}$ & $\begin{array}{c}2127.04 \pm \\
27.40^{\mathrm{b}} \\
(206)\end{array}$ & $\begin{array}{c}2.25 \pm 0.77 \\
(283)\end{array}$ \\
\hline 2011 & $\begin{array}{c}259.09 \pm \\
13.75^{\mathrm{a}} \\
(214)\end{array}$ & $\begin{array}{c}313.26 \pm 0.75 \\
(269)\end{array}$ & $\begin{array}{c}515.84 \pm \\
9.99^{\mathrm{a}} \\
(178)\end{array}$ & $\begin{array}{c}258.12 \pm \\
4.79 \\
(231)\end{array}$ & $\begin{array}{c}292.79 \pm \\
15.16 \\
(210)\end{array}$ & $\begin{array}{c}1973.90 \pm \\
30.07^{\mathrm{c}} \\
(230)\end{array}$ & $\begin{array}{c}1.73 \pm 0.08 \\
(303)\end{array}$ \\
\hline 2012 & $\begin{array}{c}261.03 \pm \\
16.75^{\mathrm{a}} \\
(140)\end{array}$ & $\begin{array}{c}313.30 \pm 0.85 \\
(187)\end{array}$ & $\begin{array}{c}499.36 \pm \\
13.56^{\mathrm{b}} \\
(104)\end{array}$ & $\begin{array}{c}274.32 \pm \\
5.37 \\
(175)\end{array}$ & $\begin{array}{c}267.27 \pm \\
18.17 \\
(131)\end{array}$ & $\begin{array}{c}2282.24 \pm \\
32.38^{\mathrm{ab}} \\
(172)\end{array}$ & $\begin{array}{c}1.62 \pm 0.09 \\
(222)\end{array}$ \\
\hline 2013 & $\begin{array}{c}247.92 \pm \\
15.21^{\mathrm{a}} \\
(159)\end{array}$ & $\begin{array}{c}312.25 \pm 0.57 \\
(223)\end{array}$ & $\begin{array}{c}524.54 \pm \\
11.13^{\mathrm{a}} \\
(137)\end{array}$ & $\begin{array}{c}265.64 \pm \\
5.02 \\
(182)\end{array}$ & $\begin{array}{c}269.43 \pm \\
17.83 \\
(146)\end{array}$ & $\begin{array}{c}2328.82 \pm \\
32.53^{\mathrm{a}} \\
(170)\end{array}$ & $\begin{array}{c}1.72 \pm 0.08 \\
(241)\end{array}$ \\
\hline 2014 & $\begin{array}{c}251.39 \pm \\
20.81^{\mathrm{a}} \\
(107)\end{array}$ & $\begin{array}{c}312.14 \pm 0.85 \\
(206)\end{array}$ & $\begin{array}{c}519.50 \pm \\
14.40^{\mathrm{a}} \\
(88)\end{array}$ & $\begin{array}{c}259.72 \pm \\
6.04 \\
(145)\end{array}$ & $\begin{array}{c}264.44 \pm \\
21.54 \\
(96)\end{array}$ & $\begin{array}{c}2232.65 \pm \\
36.99^{\mathrm{ab}} \\
(140)\end{array}$ & $\begin{array}{c}1.36 \pm 0.10 \\
(225)\end{array}$ \\
\hline 2015 & $\begin{array}{c}256.53 \pm \\
24.39^{\mathrm{a}} \\
(77)\end{array}$ & $\begin{array}{c}313.58 \pm 0.90 \\
(160)\end{array}$ & $\begin{array}{c}525.11 \pm \\
18.78^{\mathrm{a}} \\
(68)\end{array}$ & $\begin{array}{c}282.91 \pm \\
7.03 \\
(98)\end{array}$ & $\begin{array}{c}256.50 \pm \\
22.64 \\
(69)\end{array}$ & $\begin{array}{c}2359.28 \pm \\
44.42^{\mathrm{a}} \\
(98)\end{array}$ & $\begin{array}{c}1.21 \pm 0.11 \\
(166)\end{array}$ \\
\hline 2016 & $\begin{array}{c}208.29 \pm \\
18.63^{\mathrm{ab}} \\
(92)\end{array}$ & $\begin{array}{c}310.67 \pm 1.50 \\
(106)\end{array}$ & $\begin{array}{c}469.10 \pm \\
13.78^{\mathrm{b}} \\
(71)\end{array}$ & $\begin{array}{c}278.48 \pm \\
6.67 \\
(81)\end{array}$ & $\begin{array}{c}235.19 \pm \\
22.56 \\
(62)\end{array}$ & $\begin{array}{c}2422.97 \pm \\
71.96^{\mathrm{a}} \\
(81)\end{array}$ & $\begin{array}{c}1.28 \pm 0.16 \\
(109)\end{array}$ \\
\hline 2017 & $162.18 \pm$ & $311.39 \pm 1.01$ & $422.28 \pm$ & $264.42 \pm$ & $250.25 \pm$ & $2414.35 \pm$ & $1.61 \pm 0.11$ \\
\hline
\end{tabular}




\begin{tabular}{cccccccc}
\hline & $25.61^{\mathrm{b}}$ & $(109)$ & $41.57^{\mathrm{c}}$ & 10.96 & 74.53 & $61.10^{\mathrm{a}}$ & $(122)$ \\
2018 & $(51)$ & & $(11)$ & $(26)$ & $(4)$ & $(20)$ & \\
& $36.00 \pm$ & $314.60 \pm 1.49$ & $486.00 \pm$ & $261.39 \pm$ & $192.72 \pm$ & $2536.05 \pm$ & $2.22 \pm 0.20$ \\
& $22.66^{\mathrm{c}}$ & $(51)$ & $14.47^{\mathrm{ab}}$ & 3.63 & 12.67 & $64.26^{\mathrm{a}}$ & $(45)$ \\
& $(2)$ & & $(39)$ & $(95)$ & $(46)$ & $(46)$ & \\
\hline
\end{tabular}

The values with different superscript in each column differ significantly $(P \leq 0.05)$ but the values sharing at least one superscript in each column did not differ significantly $(P \leq 0.05)$.

Table 6. Effect of Season of Calving on Reproductive and Productive traits (Mean \pm SE) in Nili Ravi Buffaloes

\begin{tabular}{lccccccc}
\hline $\begin{array}{l}\text { Season of } \\
\text { Calving }\end{array}$ & $\begin{array}{l}\text { Service } \\
\text { Period }\end{array}$ & $\begin{array}{l}\text { Gestation } \\
\text { Length }\end{array}$ & $\begin{array}{l}\text { Calving } \\
\text { Interval }\end{array}$ & $\begin{array}{l}\text { Lactation } \\
\text { Length }\end{array}$ & Dry Period & $\begin{array}{l}\text { 305-day } \\
\text { Milk Yield }\end{array}$ & $\begin{array}{l}\text { Services } \\
\text { per } \\
\text { Conception }\end{array}$ \\
\hline Winter & $243.50 \pm$ & $315.64 \pm$ & $532.43 \pm$ & $275.43 \pm$ & $307.64 \pm$ & $2273.26 \pm$ & $1.78 \pm 0.09$ \\
& $21.81^{\mathrm{a}}$ & 0.71 & $11.23^{\mathrm{a}}$ & $5.16^{\mathrm{a}}$ & $17.07^{\mathrm{a}}$ & $40.85^{\mathrm{b}}$ & $(250)$ \\
Spring & $(148)$ & $(228)$ & $(118)$ & $(165)$ & $(130)$ & $(160)$ & \\
& $249.95 \pm$ & $314.85 \pm$ & $528.90 \pm$ & $258.09 \pm$ & $317.06 \pm$ & $2352.56 \pm$ & $1.71 \pm 0.08$ \\
& $22.06^{\mathrm{a}}$ & 0.79 & $11.57^{\mathrm{a}}$ & $5.57^{\mathrm{b}}$ & $19.88^{\mathrm{a}}$ & $45.67^{\mathrm{a}}$ & $(224)$ \\
Dry Summer & $(132)$ & $(194)$ & $(106)$ & $(140)$ & $(100)$ & $(135)$ & \\
& $214.37 \pm$ & $312.26 \pm$ & $483.86 \pm$ & $254.63 \pm$ & $297.92 \pm$ & $2292.42 \pm$ & $1.86 \pm 0.12$ \\
& $21.20^{\mathrm{b}}$ & 0.91 & $19.85^{\mathrm{b}}$ & $6.72^{\mathrm{b}}$ & $21.91^{\mathrm{ab}}$ & $64.45^{\mathrm{ab}}$ & $(138)$ \\
Humid & $(93)$ & $(115)$ & $(74)$ & $(71)$ & $(75)$ & $(70)$ & \\
Summer & $194.75 \pm$ & $310.22 \pm$ & $450.94 \pm$ & $263.94 \pm$ & $238.50 \pm$ & $2159.32 \pm$ & $1.48 \pm 0.05$ \\
& $8.59^{\mathrm{c}}$ & 0.49 & $14.39^{\mathrm{c}}$ & $3.35^{\mathrm{ab}}$ & $11.15^{\mathrm{b}}$ & $19.44^{\mathrm{c}}$ & $(706)$ \\
Autumn & $(464)$ & $(647)$ & $(368)$ & $(484)$ & $(407)$ & $(472)$ & \\
& $212.11 \pm$ & $311.91 \pm$ & $485.02 \pm$ & $277.77 \pm$ & $234.62 \pm$ & $2173.34 \pm$ & $1.41 \pm 0.06$ \\
& $13.24^{\mathrm{b}}$ & 0.57 & $8.31^{\mathrm{b}}$ & $3.90^{\mathrm{a}}$ & $13.20^{\mathrm{b}}$ & $26.39^{\mathrm{bc}}$ & $(398)$ \\
& $(237)$ & $(369)$ & $(189)$ & $(286)$ & $(228)$ & $(280)$ & \\
\hline
\end{tabular}

The values with different superscript in each column differ significantly $(P \leq 0.05)$ but the values sharing at least one superscript in each column did not differ significantly $(P \leq 0.05)$.

Table 7. Breeding Efficiency (\%) of Nili Ravi Buffaloes

\begin{tabular}{lcc}
\hline Category & Observations & \% age \\
\hline Buffaloes & 250 & 86.45 \\
\hline
\end{tabular}

\section{DISCUSSION}

The reproductive and productive efficiency of Nili Ravi buffaloes kept at Buffalo Research Institute (BRI), Pattoki, District Kasur, Punjab Pakistan were studied. Data concerning the reproductive and productive performance of Nili Ravi buffaloes of BRI were collected from 2010 to 2018 history sheets.

Reproductive Parameters: In this study, average age at maturity in Nili Ravi buffaloes was lower as compared to the values (1365.06 \pm 12.85 days) reported by Sule et al (2001) and to the values (1166 \pm 287.4 days) recorded by Naz and Ahmad (2006) at LPRI, Bahadurnagar, Okara. This difference might be due to difference in management and nutrition level as well as presence of good effective heat detection set up along with good teaser bulls. In this study, average age at first conception in Nili Ravi buffaloes was lower as compared to the value
$1418.6 \pm 13.16$ days presented by Sule et al (2001). This difference might be due to difference in management and nutrition level as well as good effective heat detection set up along with good teaser bulls. In this study, average age at first calving was comparatively lower the value presented by Hussain et al. (2006) where the age at first calving was $1646.87 \pm 36.31$ days in Azad Kashmir. This difference might be due to effect of adverse winter season in Azad Kashmir, difference in management, availability of green fodder and effectiveness of heat detection program. In this study, average calving interval was comparatively lower the value presented by Karim et al. (2014) where the calving interval was $547.92 \pm 10.88$ days. The lower calving interval in present study might be due to ideal number of services per conception, good management, and better feeding. The service period and gestation length are two components of calving interval. Only service period can be altered to reduce the calving interval. The data analysis of Nili Ravi buffalos revealed that average service period in this study was comparatively lower than the value presented by Naqvi and Shami (1999) in different Military farms where the service period was $280.96 \pm 9.32$ days. This difference might be due to difference in effective heat detection program along with good teaser bull and breeding 
program. On the other hand, in the study conducted in Egypt, Fooda et al. (2011b) found very short service period in Egyptian buffaloes and crossed Italian buffaloes $38.00 \pm 22.07$ days and $76.08 \pm 38.87$ days respectively. This might be result of having ideal breading system along with excellent heat detection program. The data analysis of Nili Ravi buffaloes revealed that number of services per conception was $1.60 \pm 0.29$. But Fooda et al. (2011b) found lower number of services per conception in Egyptian buffaloes $1.20 \pm 0.59$. This might be due to difference in heat detection program. The data analysis of Nili Ravi buffaloes revealed that the breeding efficiency is 86.45 percent in this study which is comparatively higher the value 82.98 percent of Murrah buffaloes in India reported by Sharma and Chaudhary (1986). This difference might be due to high genomic potential of our Nili Ravi buffaloes. This indicate that Nili Ravi buffaloes have greater capacity to reproduce in herd life. Among the reproductive traits, breeding efficiency appears to be better trait for measuring the reproductive efficiency of buffaloes.

Productive Parameters: Studies on the Nili Ravi buffaloes revealed that average 305 -day milk yield was $2211.05 \pm 20.06$ liters in this study, but Naqvi and Shami (1999) found that the average milk yield in the early maturing group buffaloes was $1912.00 \pm 12.00$ liters and late maturing group buffaloes was $1833.78 \pm 16.44$ liters, which is comparatively lower with the values found in Buffalo Research Institute, Pattoki, District Kasur in this study. One comparative study of Egyptian buffaloes, their crossing with $1 / 2$ Pakistani buffaloes and their crossing with 3/4 Pakistani buffaloes reported by Fooda et al. (2011a) found lower milk yield of $1502 \pm 344 \mathrm{~kg}, 1357 \pm$ $394 \mathrm{~kg}$ and $1383 \pm 372 \mathrm{~kg}$, respectively, which is low comparatively than present study. This difference might be due to better nutritional, availability of green fodder and good management. The data analysis of Nili Ravi buffaloes revealed that average lactation length in this study which was comparatively same the values $(266.6 \pm$ 15 days) presented by Khan and Chaudhry (2000) in Nili Ravi buffaloes maintained at Livestock Experiment Station, Bahadurnagar, Okara. Studies on the Nili Ravi buffalos at Buffalo Research Institute, Pattoki, District Kasur revealed that average dry period in this study was $279.47 \pm 5.19$ days, but Naqvi and Shami (1999) found that the average dry period in the early maturing group buffaloes was $241.59 \pm 4.18$ days and late maturing group buffaloes was $306.39 \pm 8.78$, which is comparatively lower in the early maturing group buffaloes and higher in late maturing group buffaloes with the values found in this study. One comparative study of Egyptian buffaloes reported by Aziz et al. (2001) revealing higher dry period of $307.3 \pm 51.30$ days, which was high comparatively than present study. The reason behind longer dry period might be due managemental factors and poor lactation yield.

Conclusion: It was concluded that improvement in productive and reproductive traits in Nili Ravi buffaloes can be achieved by breeding the animals with the semen of genetically superior sire, effective heat detection program, good artificial insemination, better management practices, improve nutrition, use of new reproductive technology, preventive measures, elevating the nutrition level through making silage, upgrading sanitation and ventilation system, provision of pond during summer season and providing full comfort to animal. Improved management of heifers may reduce age at maturity and age at first calving. Culling on the basis of production performance may improve productivity of buffalo in future.

Acknowledgements: I wish to express my gratitude to Prof. Dr. Khalid Javed (University of Veterinary and Animal Sciences, Lahore) for providing help in data analysis and support throughout the research work.

\section{REFERENCES}

Anonymous. (2018). Pakistan Economic Survey, Economic Affair Division, Govt. Pakistan., Islamabad.

Akhtar, M., A. Khan, T. Zaman and N. Ahmad (2006). Some clinico-epidemiological and biochemical observations of parturient haemoglobinuria in Nili-Ravi buffaloes (Bubalus bubalis). Pakistan Vet. J. 1: 1.39 .

Ali, A., K. Javed, N. Ahmad and S. Rehman (2011). Environmental factors affecting some reproductive traits in Nili Ravi buffaloes. The J. Anim. Plant Sci. 21: 868-871.

Aziz, M., S. Schoeman, G. Jordaan, O. El-Chafie and A. Mahdy (2001). Genetic and phenotypic variation of some reproductive traits in Egyptian buffalo. S. Afr. J. Anim. Sci. 31: 195-199.

Bilal, M., M. Suleman and A. Raziq. (2006). Buffalo: black gold of Pakistan. Livestock research for rural development. 18: 140-151.

Cady, R. A., S. K. Shah, E. C. Schermerhorn and R. E. McDowell (1983). Factors Affecting Performance of Nili-Ravi Buffaloes in Pakistan. J. Dairy Sci. 66: 578-586.

Chaudhry, M. A. (1992). Factors affecting the lactation length and milk yield in Nili-Ravi buffaloes. Asian Austr. J. Anim. Sci. 5: 375-382.

Elemam, M. and A.A. Nekheila (2012). Some productive traits of crossbred dairy cows in the farm of University of Khartoum, Sudan. Emir. J. Food Agric. 24: 155.

Fooda, T., A. Elbeltagi, R.H. Laila, H.S. El-habaeib and S. Awad (2011a). Evaluated of Egyptian 
buffaloes crossing with Italian buffaloes for reproductive traits. Am. J. Sci. 7: 209-213.

Fooda, T., A. Elbeltagy, R.H. Laila and S.A. El-habaeib (2011b). Assessment of Egyptian buffaloes crossing with Pakistani and Italian buffaloes for some production traits. Am. J. Sci. 7: 209-213.

Garcia, O., K. Mahmood, and T. Hemme. (2016). A review of milk production in Pakistan with particular emphasis on small-scale producers. Pro-Poor Livestock Policy Working Paper No. 3.

Hussain, Z., K. Javed, S. Hussain and G. Kiyani (2006). Reproductive performance of Nili-Ravi buffaloes in Azad Kashmir, Pakistan. The J. Anim. Plant Sci. 16: 15-19.

Karim, M., M. Hossain, M. Islam, M. Parvin and M. Matin (2014). Reproductivity, Productivity and Management System of Indigenous Buffalo (Bubalus bubalis) Cows in Costal Areas of Pirojpur and Borguna District of Bangladesh. Progressive Agric. 24: 117-122.

Khan, M. S, and H. Z. Chaudhry (2000). Lactating length and its behaviour in Nili-Ravi buffaloes. Pakistan Vet. J. 20: 81-84.
Naqvi, A. N. and S. Shami (1999). Comparative performance of early and late maturing Nili Ravi buffalo heifer. Asian Austr. J. Anim. Sci. 12: 336-340.

Naqvi, A.N. (2000). Effect of parity and season of ca lving on service period in Nili Ravi buffalo in Pakistan. Asian-Aust. J. Anim. Sci. 13(3):287291.

Naz, N. A. and M. Ahmad (2006). Genetic and phenotypic correlations for some sexual maturity traits in Nili Ravi buffalo heifers. Pakistan Vet. J. 26: 141-143.

Sharma, O. P, and R. P. Chaudhary (1986). Inheritance of breeding efficiency and its relationship with milk yield of first three lactations in buffaloes. Indian J. Anim Sci. 56: 270-273.

Sharma, O., E. Nielsen and A. N. Soerensen (1980). Views on computing breeding efficiency of buffaloes. Proc. $9^{\text {th }}$. Intl. Congr on Anim. Rep. and A.I. June 16-20, 1980.

Sule, S. R., A. L. Taparia, L.S. Jain and S.P. Tailor (2001). Reproductive status of Surti buffaloes maintained under sub-humid conditions of Rajasthan. Indian Vet. J., 78 (11): 1049-1051. 